\title{
Résultats obtenus sur les bassins expérimentaux de la France d'Outre-mer
}

\section{Results obtained from experimental basins in French Overseas Territories}

\author{
PAR M. J. RODIER \\ INGENEELR EN CHEF A ËLECTRICITÉ DE FRANCE

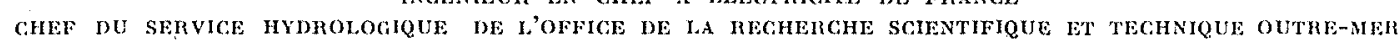

\begin{abstract}
L'emploi de nombreux bassins expérimentaux a permis d'entreprendre l'étude systématique des crues décennales en fonction des facteurs conditionnels : des résultats quantitatifs assez sirs ont été obtenus dans les domaines les plus divers: violence des crues des zones désertiques, degradation hydrographique en zone sahelienne, influence de la végétation forestière, du deboisement, rapport entre débits maxima do crues et les sols, etc.

Des données intéressantes ont été recueillies concernant l'évaporalion sur nappe d'eau libre. l'évapotranspiration et le déficil d'écoulement. Ces données sont d'accord avec celles qui résultent des relevés des stations de jaugeages classiques.
\end{abstract}

\begin{abstract}
By using a large number of experimental basins it has been possible to make a sustematic sludy of ten year floods as affected by conditional factors: reasonably reliable quantitative results were obtained concerning a large variety of subjects including the following: the violence of floods in desert regions, decreased run-off in the Sahelian region, the effect of forest cover, of deforestation, the relation befween maximum flood discharges and surface geology, ete.

Interesting data was collected concerning enaporation from open water, evapo-transpiration and retention. These data agree with data provided by conventional gauging stations.
\end{abstract}

Les données recueillies sur les premiers bassins expérimentaux des territoires d'Outre-Mer avaient fait l'objet d'une communication au Comité Technique en juin 1955. Depuis, ces études ont pris un grand développement.

En effet, pour l'ensemble des territoires et départements d'Outre-Mer, vingt-quatre bassins ont été exploités pendant la saison des pluies 1957, vingt-sept en 1958. Ces études ont été effectuées par l'Office de la Recherche Scientifique et Technique Outre-Mer ou sous sa direction. Il s'agit de climats et de sols très divers. Les fortes averses sont relativement nombreuses, de sorte qu'il a été possible de rassembler une masse de données brutes considérables. Elles n'ont été exploitées jusqu'ici que de façon partielle, surtout en vue de répondre aux demandes des Services techniques. Toutefois, cette interprétation fragmentaire a permis de tirer de très nombreux enseignements sur les méthodes de recherche les mieux appropriées et surtout sur le mécanisme du cycle de l'eau pour les différents types de climats et de sols.

\section{MODE OPERATOIRE}

Nous nous sommes orientés vers des bassins versants de $25 \mathrm{~km}^{2}$. Même pour une superficie de cet ordre, les caractéristiques du sol sont souvent hétérogènes; on exploite alors, à l'intérieur du bassin principal, un bassin de quelques $\mathrm{km}^{2}$ plus homogène. Chaque fois que cela est 
possible, on suit dans le voisinage de la station principale d'autres stations contrôlant des bassins versants de 80 à $500 \mathrm{~km}^{2}$. Enfin, on s'efforce de choisir plusieurs bassins expérimentaux pour un même type de sol et de climat, afin d'obtenir la certitude que les résultats obtenus ne correspondent pas à un cas particulier.

En moyenne, les études sur le terrain durent trois ans. On s'inspire des principes suivants:

a) Réaliser des installations légères et peu coûteuses : sections de jaugeages stabilisées par des bandes de béton, passerelles en tubes récupérables. Des déversoirs ou des venturi ne sont aménagés que si les conditions naturelles sont particulierement favorables. Par contre, chaque fois que cela est possible, il est nécessaire de barrer les zones de débordement, ce qui est facile pour de petits ruisseaux de ce genre;

b) Assurer le plein emploi de l'agent technique responsable. Pour cela, en dehors de l'aménagement du bassin, il est chargé des jaugeages, de la collecte des résultats pluviométriques, d'études d'évaporation, d'infiltration et de transport solide.

c) L'ingénieur qui dirige les études doit suivre de très près les aménagements et subir sur le bassin un certain nombre de tornades, ce qui évite des erreurs de dépouillement et d'interprétation.

Le dépouillement permel d'établir la courbe d'étalonnage et, pour chaque averse, deux documents essentiels :

- d'une part, la fiche d'averse avec son diagramme d'isohyètes donnant la répartition dans l'espace ef le hyétogramme moyen pricisant la répartition des intensités dans le temps,

et, d'autre part, l'hydrogramme brut.

L'interprétation utilise la méthode des hydrogrammes unitaires dont nous rappelons les principes essentiels. Si le ruissellement est séparé de l'écoulement hypodermique et de l'écoulement des nappes souterraines (l'hydrogramme brut étant transformé en hydrogramme de ruissellement),

- Pour toute averse de durée inférieure à une certaine limite (averse unitaire), tous les hydrogrammes de ruissellement sont des courbes affines : le temps de ruissellement restant constant, le rapport d'affinité est égal au rapport des volumes de ruissellement ou, ce qui revient au même, au rapport des hauleurs des précipitations excédentaires

- Pour deux averses unitaires successives, les ordonnées de l'hydrogramme de ruissellement résultant sont égales à la somme des ordon- nées des hydrogrammes correspondant à chacune des averses.

Il est donc possible, d'après ce qui précède,

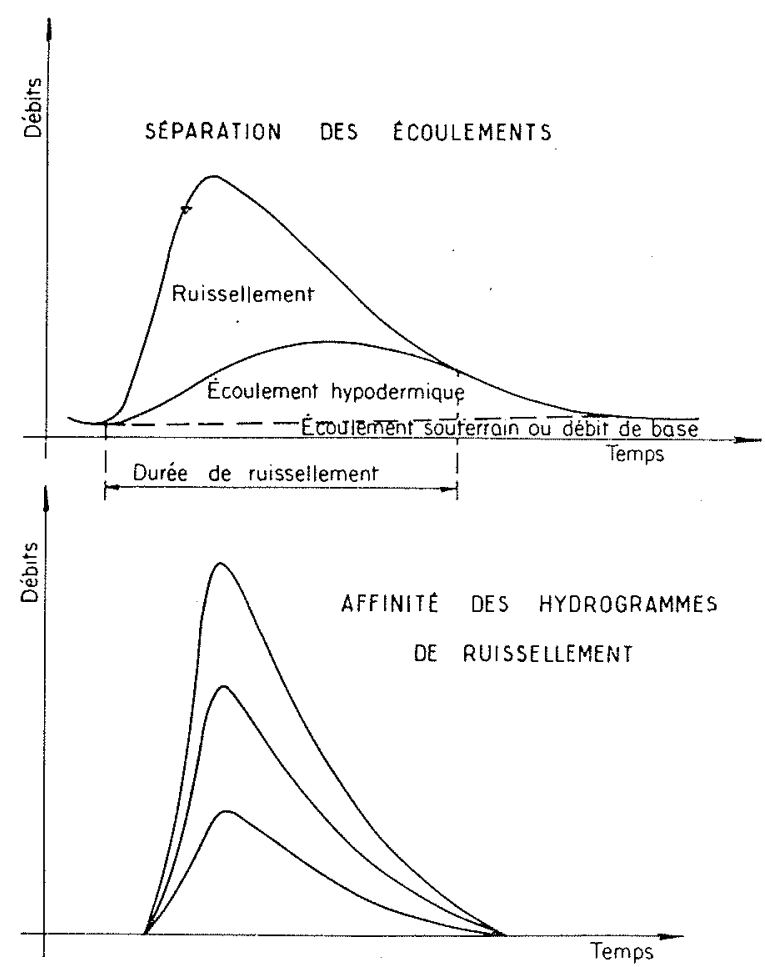

FIG. 1

Hydrogrammes unitaires.

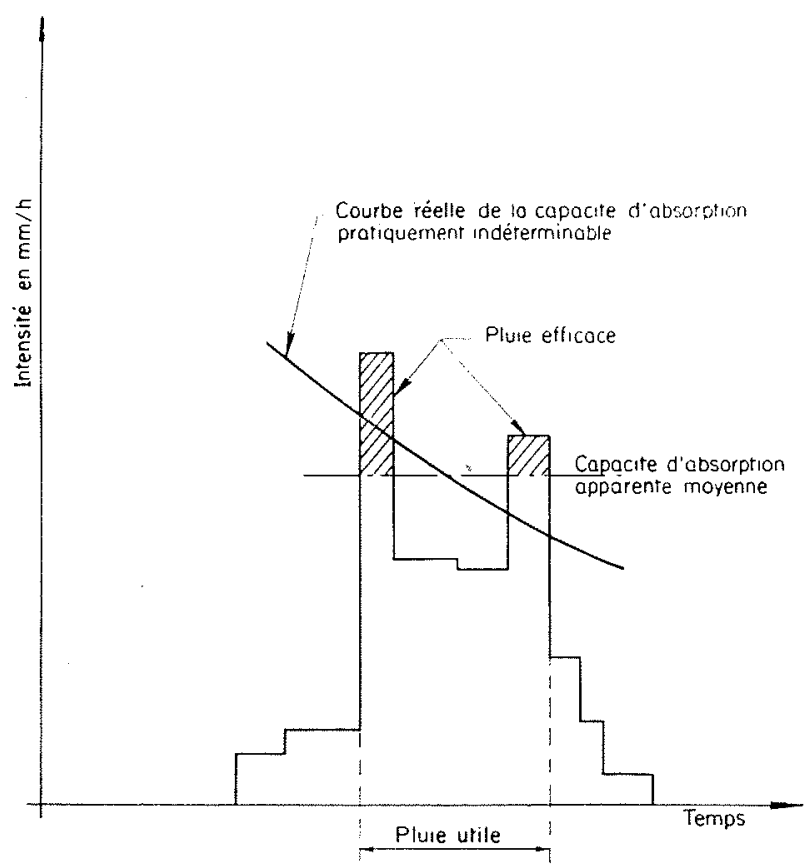

- La partie hachuree de la plure efficace représente la plule excédentarre

FIG. 2

Eléments du hyètogramme. 
de reconstituer la crue provenant d'une averse quelconque. Mais un certain nombre d'hypothèses doivent être vérifiées pour que la méthode soit applicable. En particulier, il est nécessaire que l'averse soit suffisamment homogène dans l'espace, ce qui est rarement réalisé.

D'autre part, il est difficile d'apprécier le volume de ruissellement correspondant à une hauteur donnée, ce qui exige d'estimer, soit la capacité d'infiltration ou d'absorption du sol et de sa couverture végétale, soit le coefficient de ruissellement (rapport du volume d'eau ruisselé $V_{\mathrm{R}}$ au volume des précipitations $V_{\mathrm{H}}$ ). Quoi qu'il en soit, nous avons conservé, faute de mieux, cette méthode des hydrogrammes unitaires, mais sans perdre de vue ses limites d'application, de facon à pouvoir recourir à des procédés plus sommaires chaque fois qu'elle se trouve en défaut.

Lorsque les caractéristiques hydrologicues principales ont été dégagées, on peut établir des corrélations entre ces caractéristiques et les facteurs conditionnels. C'est généralement à ce stade que les difficultés sérieuses commencent. On constate alors, même dans les cas simples des pays tropicaux, la lrès grande complexité des phénomènes auxquels s'intéressent les Hydrologues. Nous allons le voir par l'examen des résultats obtenus.

\section{RÉSULTATS CONCERNANT LES PRECIPITATIONS}

L'étude de plus de mille averses, pour des climats très diver's, a bien mis en relief l'hétérogénéité foncière de la pluie. Outre la micro-hétérogénéité à l'échelle de quelques mètres ou quelques dizaines de mètres étudiée il y a quelques années par M. Serra et l'effet perturbateur du relief quand il est suffisamment accenlué, on constate, même en terrain assez plat, que pour les averses orageuses du type tornade, la hauteur recueillie diffère sensiblement d'un pluviomètre à un autre. Les précipitations décroissent autour d'un épicentre, les isohyètes autour de ce point tracant des ovales plus ou moins déformés par le relief. On est conduit alors à définir ce que nous appelons le coefficient d'abattement: rapport entre le maximum ponctuel et la valeur moyenne sur le bassin versant, pour une averse donnée. Les variations du coefficient d'abattement avec l'importance des hauteurs de précipitations et la superficie considérée, sont très utiles à connaìtre : malheureusement, leur étude, à peine commencée, est difficile; la dispersion est très grande, comme on peut le voir par l'analyse ciapres.

Il est bien évident que le tracé de nos isohyètes correspond à une simplification extrême, mais elles nous donnent la hauteur d'eau moyenne sur le bassin avec une bonne approximation, ainsi que la position de l'épicentre, ce qui est suffisant. Nous n'insisterons pas davantage sur l'hétérogénéité dans l'espace, pour laquelle le bassin expérimental d'Alrance a fourni des données valables pour la plupart de tous nos bassins : signalons, en particulier, que le nombre minimum de postes pluviométriques nécessaires pour obtenir une bonne valeur de la moyenne est de 8 à 10, chiffre valable pour des bassins de superficie inférieure à $80-100 \mathrm{~km}^{2}$, s'ils sont régulièrement répartis.

L'examen des variations dans le temps est essentiel pour l'étude du ruissellement. Nous estimons que tout bassin expérimental doit ètre équipé d'au moins deux enregistreurs. Sur le diagramme figure 4 , nous donnons les schémas-

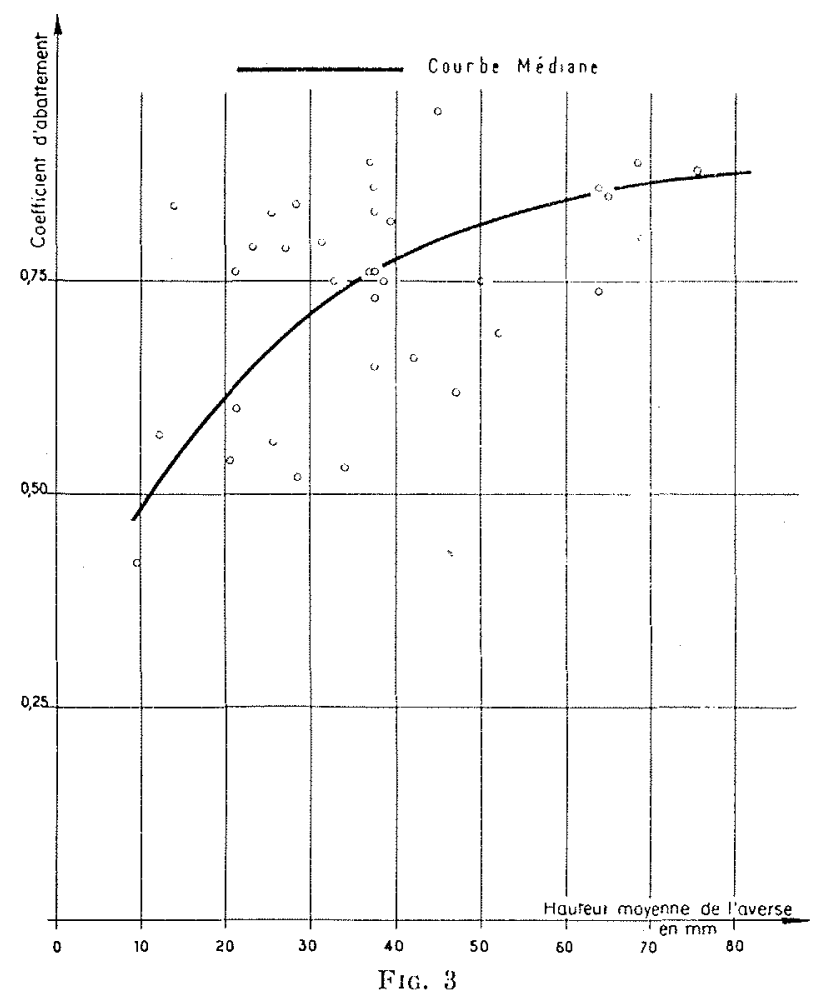

Bassin versant expérimental d'Androvakely. Variation du coefficient d'abattement en fonction de la hauteur moyenne de l'averse sur le hassin. 

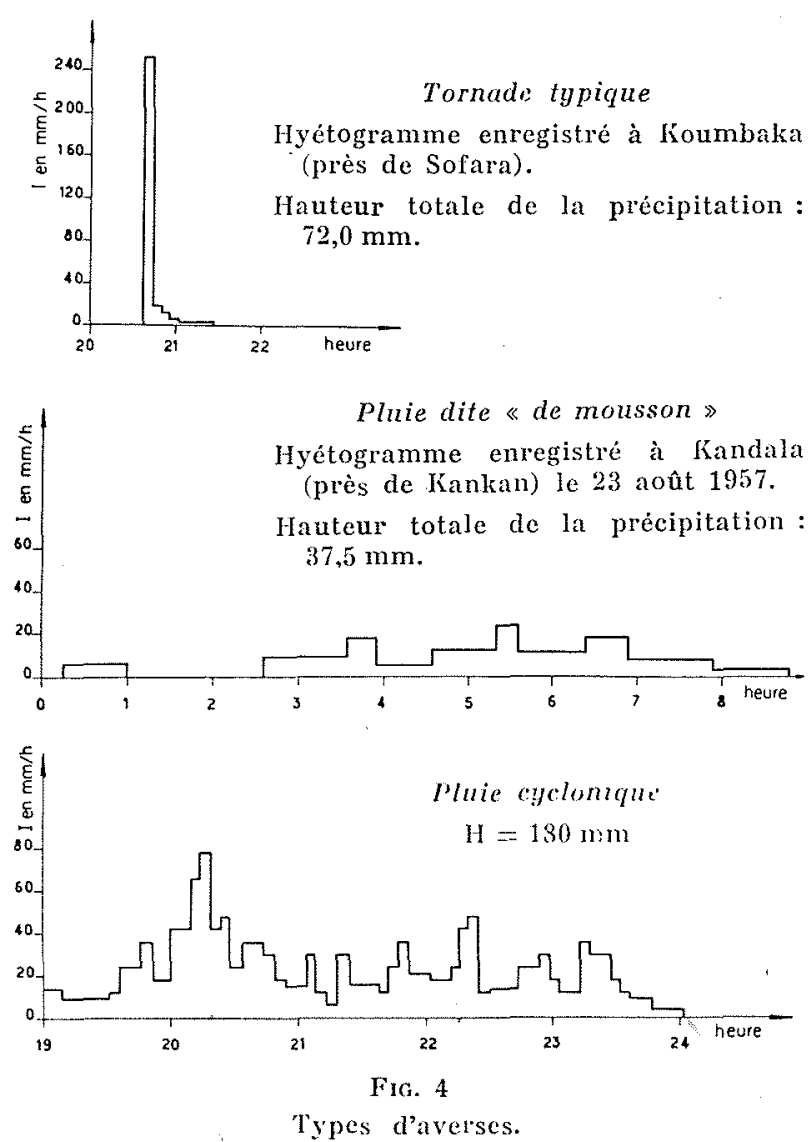

types d'averses les plus courants: tornade ou averse orageuse, «pluie de mousson » et cyclone. La forte hydraulicité de certaines années entre 1954 et 1958 , a permis l'enregistrement d'averses de fréquence assez rare, particulièrement précieuses pour les prévisions de crues. Nous avons pu constater à cette occasion, en Afrique, que pour des zones de même climat, les tornades très fortes ou les saisons des pluies déficitaires, se produisent souvent la même année.

Le nombre de pluviomètres enregistreurs des Services météorologiques étant peu nombreux et localisés au voisinage des grandes villes, les pluviographes des bassins expérimentaux, malgré la faible durée des observations, apportent un complément de documentation d'autant plus substantiel qu'il est possible d'étudier également la répartition dans l'espace de ces averses. Dans deux ou trois ans, il sera possible de multiplier le nombre de courbes intensité-durée, encore beaucoup trop réduit.

Enfin, nous avons essayé, dans certains cas, de multiplier les enregistreurs sur un même bassin. On trouvera, ci-après, une série de diagrammes correspondant à l'enregistrement d'une même averse en six points d'un bassin versant de $29,4 \mathrm{~km}^{2}$. Les diagrammes ont tous un même air de famille et on conçoit qu'avec deux enregistreurs on puisse, en général, imaginer un dia- gramme moyen susceptible de s'appliquer, sans trop de risques d'erreurs, à l'ensemble du bassin; mais il semble inutile de poursuivre la recherche de méthodes raffinées pour l'utilisation des données des pluviographes. Il est en effet nécessaire, pour l'interprétation, de mettre au point une représentation simple et utilisable d'une averse donnée, pour l'ensemble du bassin. La méthode de Horton correspond théoriquement à la solution idéale, mais elle repose sur des hypothèses rarement réalisées.

Généralement, nous supposons que le bassin a subi une averse de hauteur constante avec un

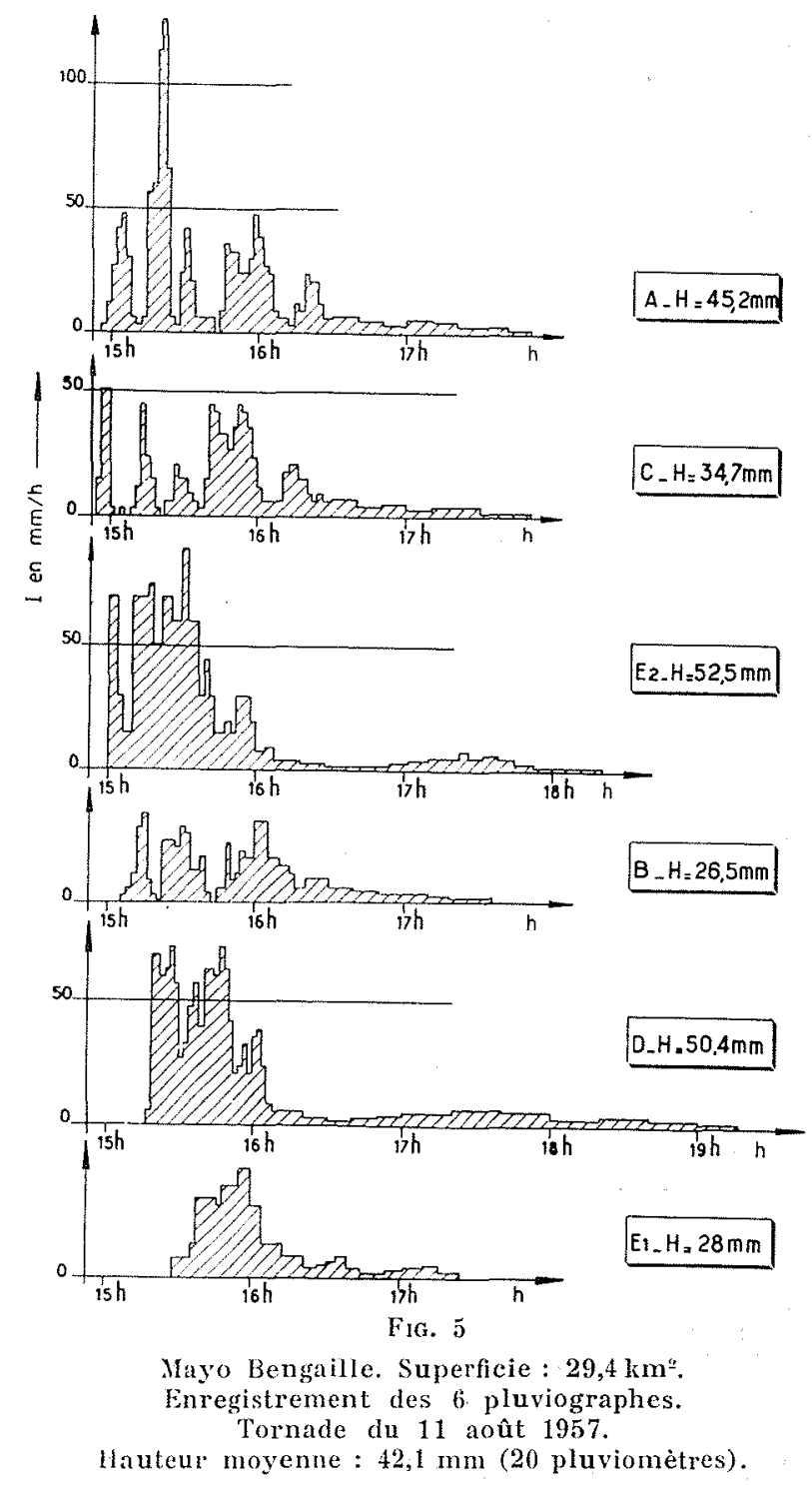

diagramme d'intensité, identique en tous les points. Pour l'établissement de ce hyétogramme moyen, plusieurs méthodes ont été mises au point, suivant les divers cas rencontrés.

La recherche de corrélation entre les caractéristiques de la crue et les données simples telle que intensité maximum ou intensité moyenne ou 
hauteur globale, est souvent décevante et ceci explique que bien souvent les chercheurs aient été découragés. Mais il est souvent possible, grâce à l'observation directe des phénomènes, de trouver le moyen de tourner ces difficultés. Nous citerons deux exemples: sur les bassins de forêt équatoriale, ce qui conditionne le ruissellement c'est la pluie telle qu'elle tombe sur le sol et non telle qu'elle arrive sur la forêt, ou, ce qui revient à peu près au même, telle qu'elle est enregistrée par le pluviographe. Il y a là un phénomène de régularisation. La capacité d'absorption résultant des diagrammes bruts des pluviographes serait beaucoup trop forte, ce serait presque une fonction directe de l'intensité maximum. Il faut considérer des diagrammes établis en prenant des intervalles assez grands à intensité constante, dix minutes au moins. On peut même dans de nombreux cas utiliser, pour les corrélations, la hauteur de la pluie pour toutes les intensités supérieures à des valeurs assez faibles, 5 à $10 \mathrm{~mm} /$ heure.
Dans toutes les autres régions, une notion s'impose, celle de pluie utile ou de pluie efficace : au point de vue du ruissellement, seules comptent les fractions de l'averse à moyenne ou forte intensité. On cherche donc à isoler dans le hyétogramme la fraction qui donnerait le mème ruissellement que celui qui a été réellement observé. La pluie efficace correspond sur le hyétogramme à la période pendant laquelle il y a eu ruissellement. Cette définition est précise, mais trop artificielle. Dans la plupart des cas, la pluie efficace donnerait un volume de ruissellement bien inférieur à celui de l'hydrogramme réel. C'est pourquoi on lui a substitué la notion de pluie utile, plus concrète, mais plus vague. Elle correspond à la partie essentielle de l'averse, c'est la fraction du hyétogramme correspondant à des intensités supérieures à un seuil donné, 10, 15, $20 \mathrm{~mm} / \mathrm{h}$, variable suivant la perméabilité du sol et la densité de la végétation et très inférieure à la capacité d'absorption.

\section{RESULTATS CONCERNANT LES DÉBITS DE CRUES. - RUISSELLEMENT CAPACITE D'ABSORPTION}

Sur les petits bassins des régions tropicales et équatoriales, les fortes crues sont produites par le ruissellement. Ce dernier est souvent violent.

La détermination d'une crue de fréquence donnée, crue décennale par exemple, à partir de l'averse correspondante, suppose la détermination de l'hydrogramme-type ou diagramme de distribution et du coefficient de ruissellement ou de la capacité d'absorption évaluée en $\mathrm{mm} / \mathrm{h}$. En soustrayant cette capacité des ordonnées du hyétogramme, également en $\mathrm{mm} / \mathrm{h}$, on obtient l'intensité excédentaire $H_{e x c}$ responsable du ruissellement. Le volume de ruissellement étant donné par :

$$
\mathrm{V}_{\mathrm{R}}=\mathrm{H}_{\mathrm{exe}} \times \mathrm{S}
$$

L'emploi de la capacité d'absorption est séduisant; en pratique, il peut conduire à des erreurs graves. Il s'agit, en fait, de tronquer un diagramme par une ordonnée égale à cette capacité d'absorption, la capacité d'absorption n'étant pas très inférieure aux intensités maxima du hyétogramme. On conçoit qu'une faible erreur sur la position de cette ordonnée peut avoir des répercussions beaucoup plus fortes sur la valeur de la surface excédentaire, donc du volume de ruissellement, que celle qui résulterait de l'emploi d'un coefficient de ruissellement peu pré- cis, car ce dernier est ulilisé comme un rapport et non comme une différence.

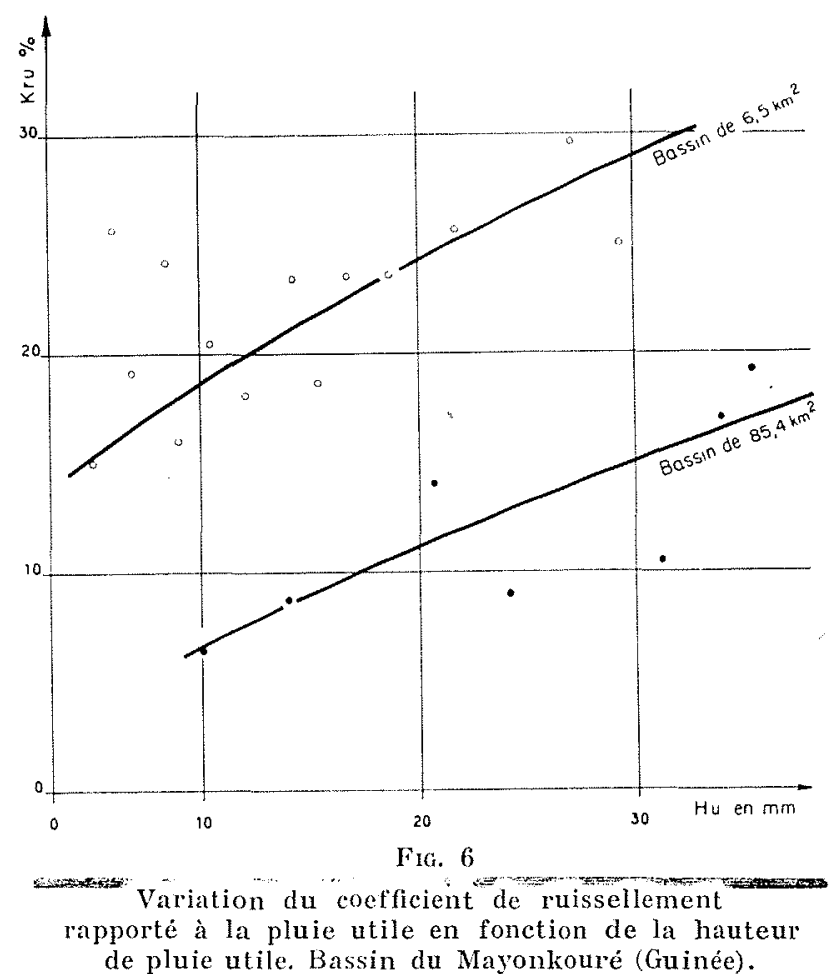


L'estimation, a priori, du coefficient de ruissellement pour une averse donnée est délicate. En effet, sur un même bassin, ce coefficient est fonction de la hauteur d'eau précipitée, de l'intensité maximum de l'averse, ou plus exactement de la forme du hyétogramme, de l'état de saturation du sol et, souvent, de l'état de la couverture végétale, c'est-à-dire de la saison. Suivant le type de climat, les facteurs prédominants diffèrent : vers le désert, par exemple, c'est l'intensité moyenne de la précipitation orageuse; en forêt, ce serait au contraire la hauteur de l'averse. On est réduit à étudier le coefficient de ruissellement en fonction d'un ou deux de ces facteurs, les autres étant supposés constants. En fait, ils ne le sont pas, ce qui introduit une forte dispersion, comme on peut le voir sur la figure 6 . La notion du coefficient de ruissellement utile réduit, dans une certaine mesure, cette dispersion, ainsi que l'utilisation d'un indice de saturation.

Le nuage de points obtenu en portant les valeurs du coefficient de ruissellement en fonction de la hauteur de pluie utile, par exemple, n'est pas très encourageant. Mais, si l'on porte sur le même graphique les nuages de points correspondant à des bassins différents, pour des averses de type analogue, l'ensemble met en évidence des tendances bien marquées pour chacun de ces bassins. Il est possible, par exemple, de donner pour l'averse décennale des ordres de grandeur du coefficient de ruissellement, done de calculer les crues décennales.

Le calcul de ces crues présente un intérèt incontestable, c'est même souvent l'objet principal de nos contrats d'études; en outre, les chifres de débit maximal auxquels nous aboutissons, donnent une idée de la susceptibilité au ruissellement des bassins, en attendant qu'un indice simple, correspondant à cette notion, soit défini. En effet, pour la majeure partie de l'A.O.F., de l'A.E.F. et du Cameroun, les averses décennales varient de 90 à $120 \mathrm{~mm}$; les bassins sont donc soumis, dans ce cas, à des averses assez voisines (sauf les bassins proches du désert et ceux voisins du littoral).

Nous avons entrepris, depuis plusieur's années, l'écude systématique de ces crues décennales et nous mettons patiemment au point un tableau général qui est complété au fur et à mesure de l'avancement de nos études.

Les valeurs des débits spécifiques de crues ont été ramenées, par correction, à une superficie de $25 \mathrm{~km}^{2}$. 'Théoriquement, les débits spécifiques suivent une formule du genre de :

$$
q=\mathrm{AS}^{n-1} \text { en } 1 / \mathrm{s} . \mathrm{km}^{2} \text { ou } \mathrm{Q}=\mathrm{AS}^{n} \text { en } \mathrm{m}^{3} / \mathrm{s}
$$

La correction n'est pas très délicate tant que les superficies sont comprises entre 10 et $50 \mathrm{~km}^{2}$. Les pentes ne sont indiquées que de façon quali- tative : très fortes $>5 \%$, fortes 2 à $5 \%$, assez fortes 1 à $2 \%$.

Lorsque ce tableau se sera suffisamment développé, il sera possible de considérer isolément l'influence de l'un des facteurs du ruissellement faisant l'objet de chaque colonne, en supposant que tous les autres facteurs restent les mêmes. Nous n'en sommes pas encore là; cependant, il est possible de donner plus que des indications qualitatives.

On voit que l'influence du sol est très grande si la couverture végétale est assez peu épaisse, alors que celle du sous-sol est très faible. Le même grès peu perméable donne lieu à des crues de 8 à $10000 \mathrm{l} / \mathrm{s} . \mathrm{km}^{2}$ sous l'isohyète 600 , alors que, pour une averse plus forte, il ne produira que $1500-1800 \mathrm{l} / \mathrm{s}, \mathrm{km}^{2}$ s'il est recouvert de latérite très fissurée.

L'influence très forte de la végétation est bien mise en évidence. Si nous prenons les deux extrèmes, un bassin du désert, pour une averse de $60 \mathrm{~mm}$, donne lieu à un débit quatre fois plus fort qu'un bassin de forêt de même pente, qui recoit, cependant, une averse de hauteur deux fois plus grande.

Deux faits viennent confirmer cette tendance :

- La forte valeur des débits de crue en cité africaine. Pour Brazzaville, le sol dénudé, plus ou moins compacté par le piétinement, doit permettre des débits de crues décennales quatre à cinq fois plus élevés que le sable recouvert de graminées qui constitue le terrain naturel.

- La décroissance des débit de crues au cours de la saison des pluies, avec la croissance de la végétation.

Une certaine imperméabilité du sol, à la première pluie de la saison, a pu également être mise en évidence au nord de l'isohyète 800 . Elle passe souvent inaperçue, car cette première tornade est généralement de faible importance.

En région forestière, le ruissellement est faible. En fait, il s'agit d'un phénomène d'écoulement mal défini, nettement différent du ruissellement que l'on observe plus au nord. Les débits de crues décennales, compris entre 400 et $1000 \mathrm{l} / \mathrm{s} . \mathrm{km}^{2}$, varient peu avec la pente, tout au moins si le relief n'est pas vigoureux. Mais, si la pente s'accentue, les débits augmentent nettement, atteignant 1000 et peut-être 3 à 4000 $\mathrm{l} / \mathrm{s} . \mathrm{km}^{2}$, si la pente devient très forte (de l'ordre de $45^{\circ}$ ).

De façon générale, l'influence du sol est beaucoup moins grande en forêt qu'en savane.

On a cherché également à étudier la décroissance de la capacité d'absorption ou la croissance du coefficient de ruissellement au cours de l'averse. Deux faits semblent intéressants : pour 
GRUES DÉCENNALES SUR QUELQUES BASSINS TYPES D'AFRIQUE NOIRE

$\left(\right.$ Superficie $\left.=25 \mathrm{~km}^{2}\right)$

\begin{tabular}{|c|c|c|c|c|c|c|c|}
\hline$N^{0}$ & $\begin{array}{c}\text { Régime } \\
\text { hydrologique }\end{array}$ & Pente & Sous-sol & Sol & Végétation & $\begin{array}{c}\text { Précipitations } \\
\text { annuelles } \\
\text { en mm }\end{array}$ & $\begin{array}{c}\text { Crue décennale } \\
\qquad 1 / \mathrm{s} \cdot \mathrm{km}^{2}\end{array}$ \\
\hline 1 & Désertique & Forte & $\begin{array}{l}\text { Grès } \\
\text { imperméable }\end{array}$ & Inexistant & Rare & 100 & 4 à 6000 \\
\hline 2 & $\begin{array}{l}\text { Presque } \\
\text { subdésertique }\end{array}$ & $\begin{array}{l}\text { Assez } \\
\text { forte }\end{array}$ & Quartzites & $\begin{array}{l}\text { Assez } \\
\text { imperméable }\end{array}$ & Clairsemée & 300 & 3500 \\
\hline 3 & $\begin{array}{l}\text { Presque } \\
\text { subdésertique }\end{array}$ & Modérée & Quartzites & $\begin{array}{l}\text { Assez } \\
\text { imperméable }\end{array}$ & $\begin{array}{l}\text { Tres } \\
\text { clairsemée }\end{array}$ & 300 & 2000 \\
\hline 4 & Sabélien & $\begin{array}{l}\text { Assez } \\
\text { forte }\end{array}$ & Grès & $\begin{array}{l}\text { Pratiquement } \\
\text { inexistant }\end{array}$ & Clairsemée & 600 & 8000 à 10000 \\
\hline 5 & Sahélien & Forte & $\begin{array}{l}\text { Grès } \\
\text { ferrugineux }\end{array}$ & $\begin{array}{l}\text { Argileux } \\
\text { imperméable }\end{array}$ & $\begin{array}{l}\text { Clairsemée } \\
\text { cultures }\end{array}$ & 500 & 7000 à 8000 \\
\hline 6 & Sahélien & $\begin{array}{l}\text { Assez } \\
\text { forte }\end{array}$ & $\begin{array}{l}\text { Grès } \\
\text { ferrugineux }\end{array}$ & $\begin{array}{l}\text { Même terrain } \\
+ \text { latérite }\end{array}$ & Clairsemée & 500 & 4000 \\
\hline 7 & Sahélien & Faible & Granitique & $\begin{array}{l}\text { Argileux très } \\
\text { imperméable }\end{array}$ & Graminées & 450 & 1600 à 1700 \\
\hline 8 & Sahélièn & $\begin{array}{l}\text { Assez } \\
\text { forte }\end{array}$ & Graniticue & $\begin{array}{l}\text { Légèrement } \\
\text { perméable }\end{array}$ & Clairsemée & 450 & 1500 \\
\hline 9 & Tropical & $\begin{array}{l}\text { Très } \\
\text { forte }\end{array}$ & Andésite & Imperméable & Clair'semée & 800 & 8000 à 10000 \\
\hline 10 & Tropical & Forte & $\begin{array}{l}\text { Grès } \\
\text { imperméable }\end{array}$ & $\begin{array}{l}\text { Latérite très } \\
\text { perméable }\end{array}$ & Savane claire & 1000 & 1500 à 1800 \\
\hline 11 & $\begin{array}{l}\text { Tropical de } \\
\text { transition }\end{array}$ & Forte & Grès & $\begin{array}{l}\text { Perméabilité } \\
\text { moyenne }\end{array}$ & Savane boisée & 2100 & 2000 \\
\hline 13 & $\begin{array}{l}\text { Tropical de } \\
\text { transition }\end{array}$ & Forte & Quartzile & $\begin{array}{l}\text { Perméable } \\
\text { sablonneux }\end{array}$ & $\begin{array}{l}\text { Savane } \\
\text { boisée dense }\end{array}$ & 1600 & 400 à 500 \\
\hline 14 & Equatorial & $\begin{array}{l}\text { Assez } \\
\text { forte }\end{array}$ & $\begin{array}{l}\text { Schisto- } \\
\text { gréseux }\end{array}$ & Imperméable & $\begin{array}{l}\text { Savane } \\
\text { (pseudo steppe) }\end{array}$ & 1400 & 8000 à 9000 \\
\hline 15 & Equatorial & $\begin{array}{l}\text { Assez } \\
\text { forte }\end{array}$ & Sable & Imperméable & $\begin{array}{l}\text { Cité } \\
\text { africaine }\end{array}$ & 1500 & 4000 à 6000 \\
\hline 16 & Equatorial & Forte & Gneiss & Imperméable & Forêt & 1800 & 400 à 600 \\
\hline 17 & Equatorial & Modérée & $\begin{array}{l}\text { Granito- } \\
\text { gneiss }\end{array}$ & $\begin{array}{l}\text { Assez } \\
\text { perméable }\end{array}$ & Forêt & 1300 & 400 a 600 \\
\hline 18 & Equatorial & Forte & $\begin{array}{l}\text { Granito- } \\
\text { gneiss }\end{array}$ & $\begin{array}{l}\text { Assez } \\
\text { perméable }\end{array}$ & $\begin{array}{l}\text { Forêt } \\
\text { dégradèe }\end{array}$ & 1800 & 1500 a 2000 \\
\hline
\end{tabular}


des averses faibles ou moyennes, la capacité d'absorption du début de l'averse croit avec l'intensité maximale jusqu'à une certaine limite, at-

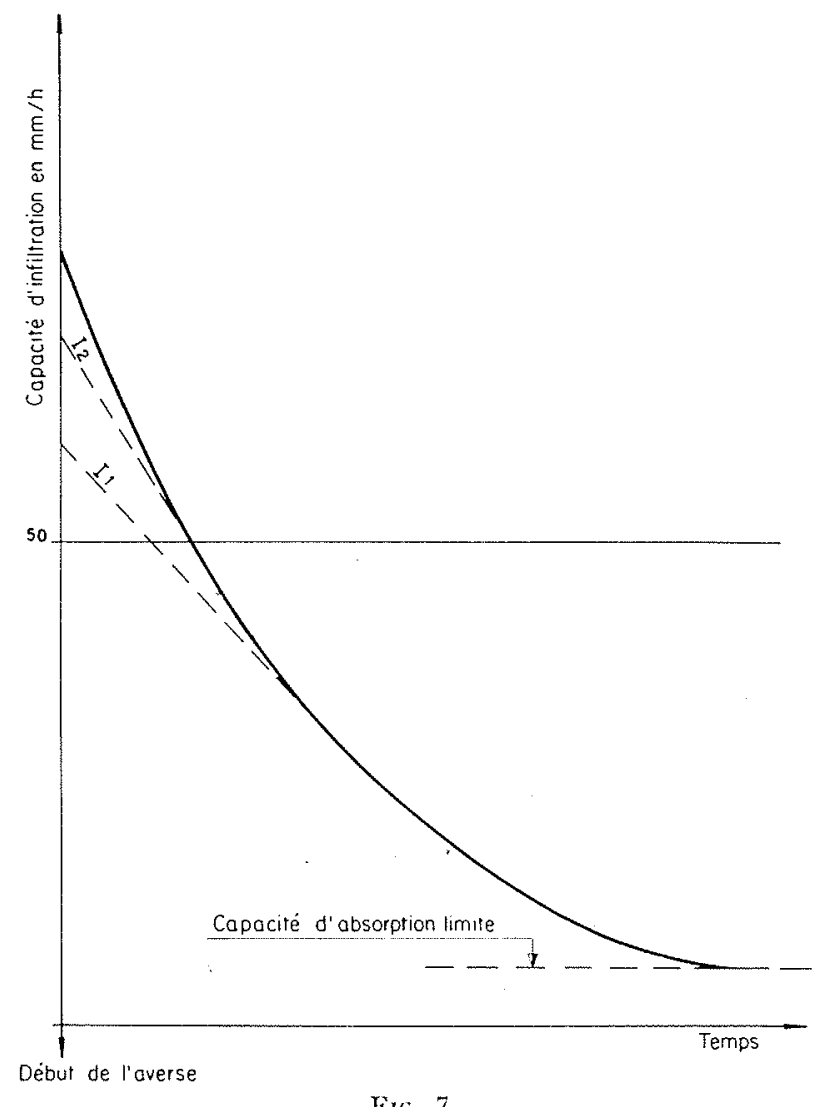

Fric. 7

Variation de la capacite d'absorption an cours d'une arerse. teinte soit pour de très fortes intensités, soit pour des averses très prolongées, alors que, théoriquement, elle devrait être constante. Ceci tient au fait que les bassins versants sont rarement homogènes, la fraction de la superficie qui ruisselle croît avec l'intensité ou la durée de l'averse.

D'autre part, en fin d'averses fortes et très prolongées, on a trouvé que la capacité d'absorption présentait, pour des sols et des climats très divers, des valeurs limites analogues de 4 à $6 \mathrm{~mm} / \mathrm{h}$. Traduite en débit spécifique (1500 $\left.1 / \mathrm{s}^{2} \mathrm{~km}^{2}\right)$, cette valeur semble énorme, mais n'oublions pas que, si l'intensité excédentaire correspond au ruissellement, ces 4 à $6 \mathrm{~mm} / \mathrm{h}$ correspondent au débit hypodermique et au débit permanent des nappes.

Une valeur fréquente de la capacité d'absorption au début d'une averse, en savane et en région sahélienne, est de 35 à $45 \mathrm{~mm} / \mathrm{h}$. Les régions forestieres donnent des valeurs beaucoup plus fortes, mais leur signification physique n'est pas toujours bien nette, comme nous l'avons vu plus haut.

En zones désertique el subdésertique, les études ont mis en évidence les très forts volumes d'eau produits dans les zones accidentées, par des averses orageuses relativement faibles (8 à $15 \mathrm{~mm}$ ).

Pour en terminer avec lc ruissellement, précisons que l'étude des temps de réponse (lag) des bassins a permis de trouver qu'en savane, la vilesse de ruissellement est de l'ordre de 10 à $20 \mathrm{~cm} / \mathrm{s}$ sur le sol et de l'ordre de $0,50 \mathrm{~m}$ à $1 \mathrm{~m}$ dans le réseau hydrographique, sans que nous ayons eu le temps de procéder à des études vaiment systématiques sur cette question.

\section{RESULTATS CONCERNANT L'INFILTRATION}

L'infiltration a fait l'objet de mesures directes, par la méthode de Muntz ou la méthode Porcher, en liaison avec la détermination du bilan hydrologique de chaque averse.

L'interprétation de ces mesures est à peine commencée. Les résultats sont affectés d'une très grande dispersion, mais, comme nous l'avons vu plus haut, la comparaison des nuages de points est instructive. Par exemple, un terrain d'arène granitique du Dahomey donne des résultats Muntz variant de 150 à $600 \mathrm{~mm} / \mathrm{h}$, alors qu'un terrain argileux du Nord Cameroun donne de 20 à $80 \mathrm{~mm} / \mathrm{h}$. Ces derniers chiffres étant confirmés par les bilans hydrologiques.

Des études intéressantes ont également été faites pour déterminer l'apparition de l'écoulement superficiel.
La figure 8 représente, pour un bassin de perméabilité moyenne (arène granitique) situé sous l'isohyète 550 et recouvert d'une végétation réduite, la courbe précisant la hauteur de pluie limite donnant lieu à ruissellement en fonction de l'intervalle avec l'averse précédente. La durée de l'averse joue peu; il s'agit toujours de courtes tornades. Les points aberrants sont assez rares, ils correspondent précisément à des caractéristiques d'intensité anormales.

Pour le graphique figure 9 , en zone guinéenne avec couverture végétale assez épaisse et pluie de mousson, la courbe limite a été représentée en fonction de la durée de l'averse; le degré de saturation du terrain variant peu, l'intervalle de temps entre deux averses est sans grande influence. 


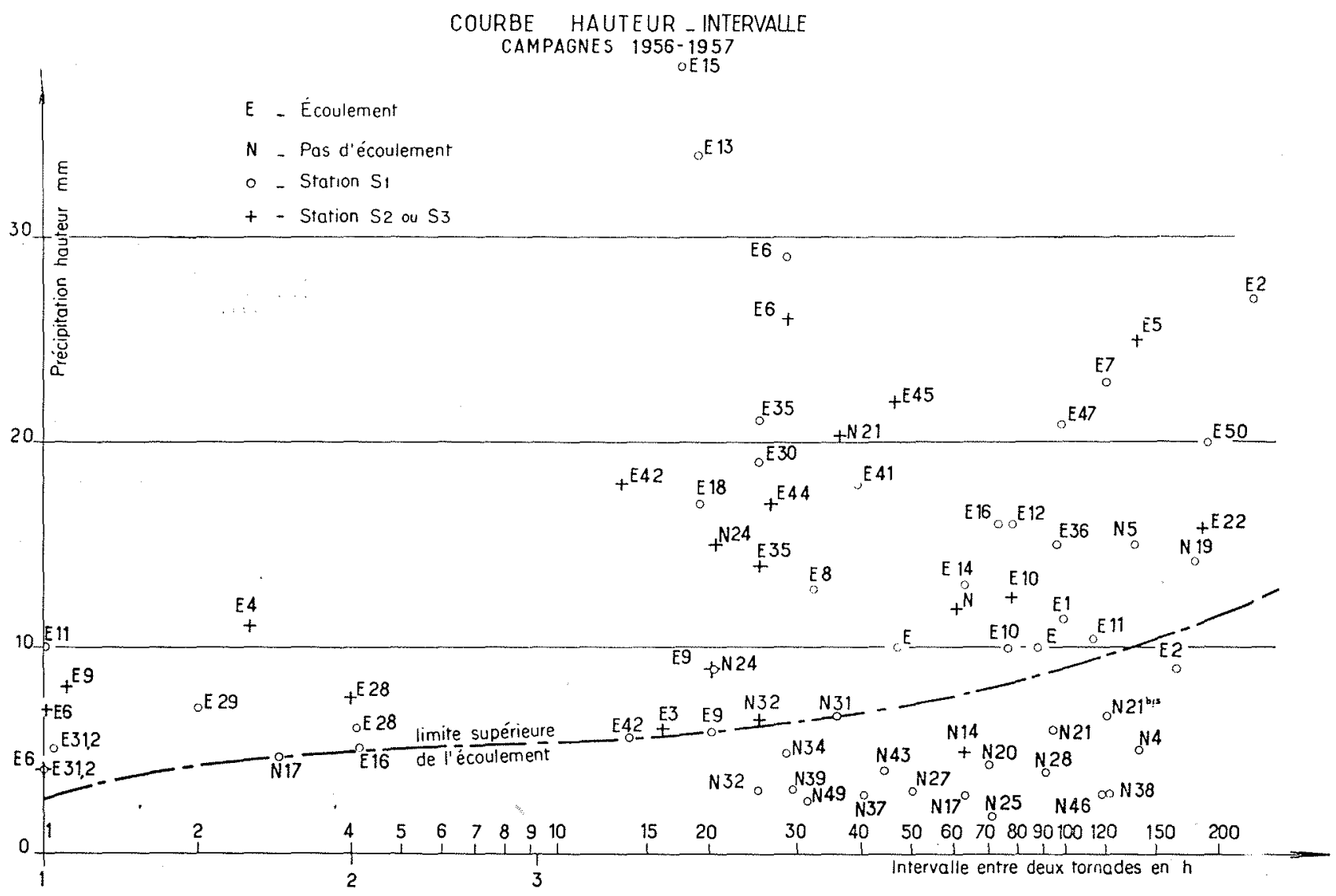

FIG. 8

Ouadi Kaoun. Recherche de la précipitation limite de ruissellement.

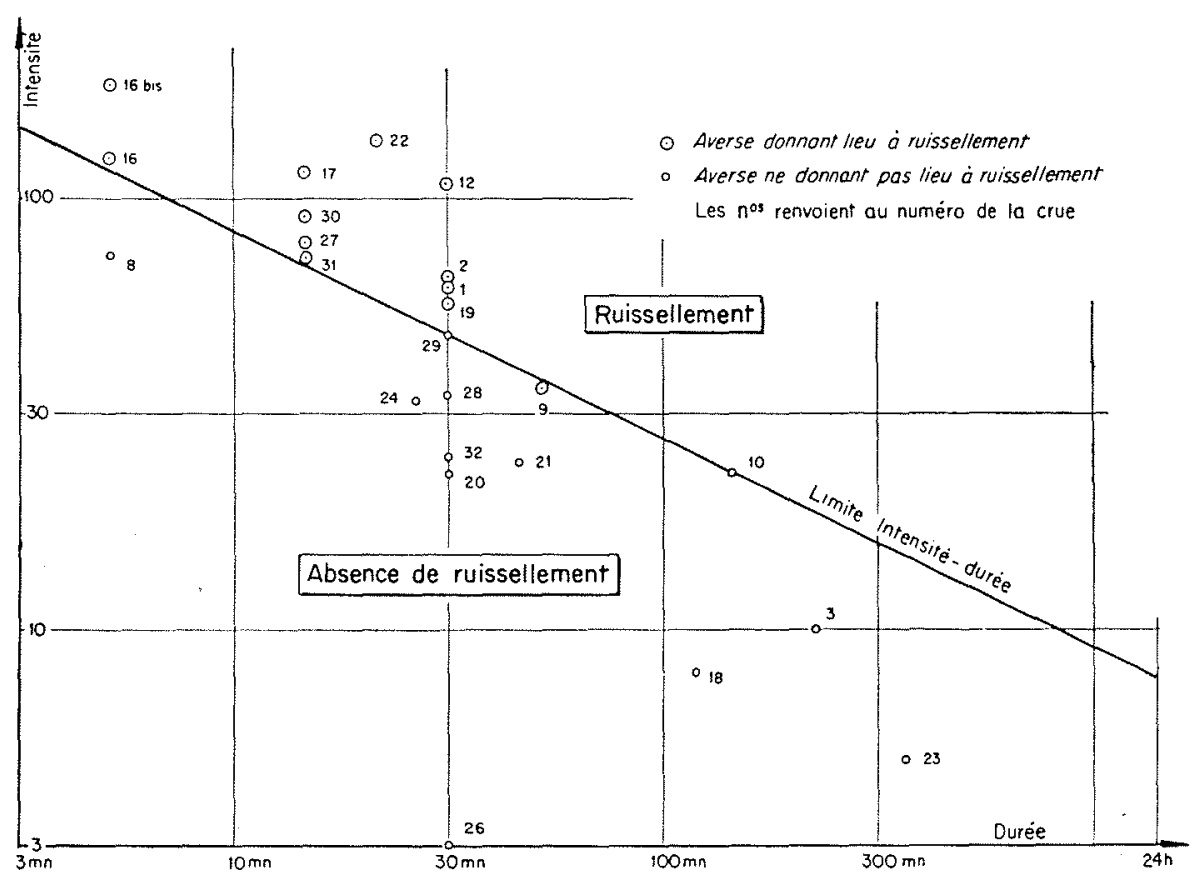

FIG. 9 
Si on considère des valeurs courantes de l'intervalle et de la durée de l'averse, on voit que, dans le premier cas, une averse de $8 \mathrm{~mm}$ donne lieu à écoulement, alors que $30 \mathrm{~mm}$ semblent nécessaires dans le second.

\section{RESULTATS CONCERNANT L'EVAPORATION - L'EVAPOTRANSPIRATION LE DEFICIT D'ÉCOULEMENT}

Les stations évaporométriques installées sur les bassins ont étendu nos connaissances sur l'évaporation des nappes d'eau libre; mais l'exposé des résultats obtenus dans ce domaine nous entraînerait trop loin. Nous nous bornerons à l'évaporation effective et potentielle et au déficit d'écoulement.

Les études sur bassins expérimentaux devraient apporter, a p̧̄iori, des données intéressantes dans ce domaine. En réalité, là encore, la complexité des phénomènes conduit à des difficultés non négligeables. Les phénomènes de rétention, beaucoup moins importants que sur les grands bassins, sont encore notables. En général, l'évapotranspiration effective varie dans de très grandes proportions d'un jour à l'autre.

Nous examinerons deux cas relativement sim-
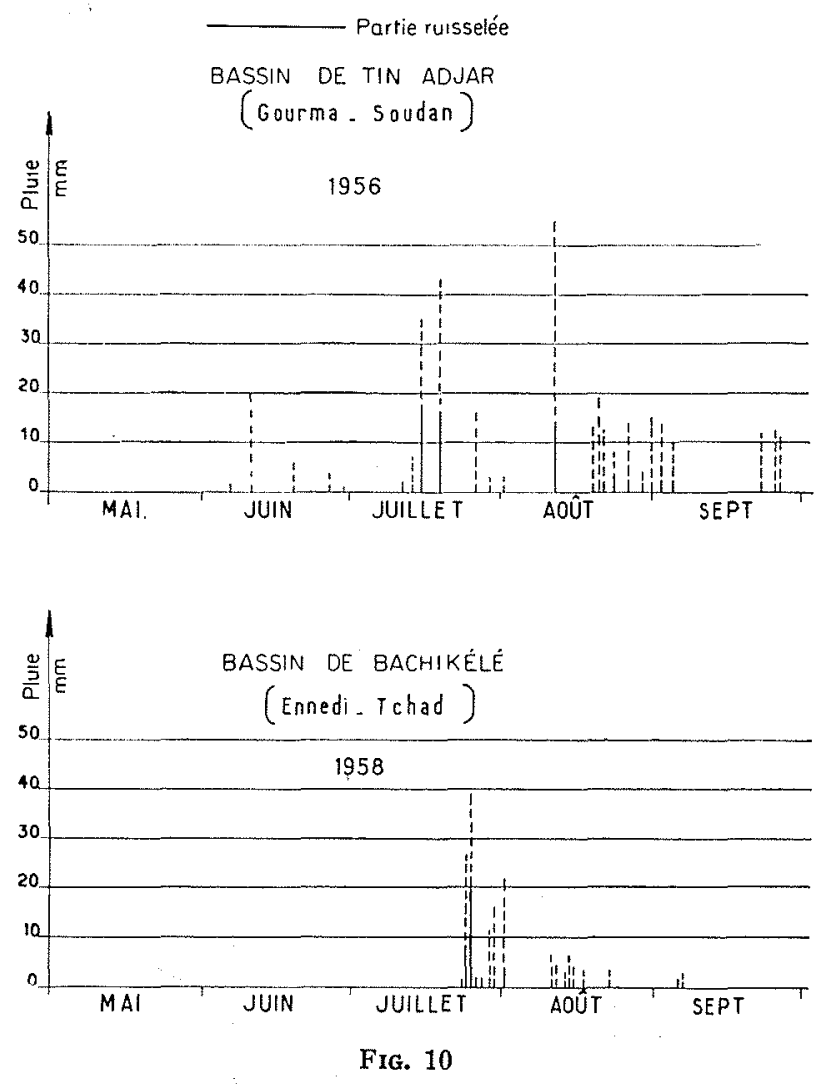

Déficit d'écoulement journalier. ples: un bassin subdésertique, celui de Tin Adjar, et un bassin de zone guinéenne ou forestière.

Sur les bassins subdésertiques, l'eau apportée par les averses a pratiquement disparu au bout de deux ou trois jours. Il n'y a pratiquement pas d'inféroflux. La majeure partie de l'eau qui n'a pas rejoint le réseau hydrographique s'évapore en vingt-quatre heures.

L'évapotranspiration est étroitement liée aux précipitations; le coefficient d'écoulement annuel est faible, il dépend beaucoup plus de l'importance des diverses averses et de leur répartition que de la hauteur d'eau mensuelle ou annuelle. L'évapotranspiration potentielle, valeur limite, serait peut-être égale à $15 \mathrm{~mm}$ à Tin Adjar.

Un autre cas est celui de la zone guinéenne ou des régions forestières. La couverture végétale y est dense et, pendant de longues périodes, le sol est saturé presque en permanence. L'évapotranspiration devrait donc se rapprocher de l'évapotranspiration potentielle. La rétention est très importante et les premiers mois de saison des pluies accusent des déficits d'écoulement exagérés; mais, en fin de saison, si les précipitations se maintiennent, le déficit d'écoulement doit être voisin de l'évapotranspiration. Les résultats du Leyou (frontière Moyen-Congo-Gabon) nous montrent que l'évapotranspiration serait de $4 \mathrm{~mm} /$ jour, supérieure à l'évapotranspiration sur bac Colorado : $3 \mathrm{~mm} /$ jour. Ce fait est confirmé par la comparaison des déficits d'écoulement annuels sur grand bàssin. Sur le Nion (Côte d'Ivoire), on retrouve un chiffre de 3,5 à $5 \mathrm{~mm}$. Tous ces résultats sont légèrement supérieurs à l'évaporation sur bac Colorado trouvée sur les mêmes hassins. Une étude systématique, averse par averse, permettrait certainement de dégager des chiffres plus sûr's et de mieux suivre les variations d'un climat à un autre ou d'un sol à un autre.

Un dernier exemple intéressant est celui d'Androvakely sur les Hauts-Plateaux de Madagascar. Nous y avons observé des bacs Colorado et des lysimètres, l'un normal et l'autre utilisé comme une cuve de Thornthwaite, donnant directement l'évapotranspiration potentielle. 
BILAN HYDROLOGIOUE DU BASSIN D'ANDROVAKELY

(Hauteur en $\mathrm{mm}$ )

\begin{tabular}{|c|c|c|c|c|c|c|c|c|c|c|c|c|c|c|}
\hline \multirow{6}{*}{ 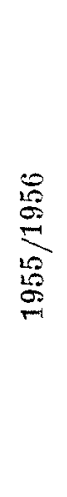 } & & $\mathrm{N}$ & $\mathrm{D}$ & $\mathrm{J}$ & $\mathrm{F}$ & $M$ & A & $M$ & $\mathrm{~J}$ & J & $A$ & S & o & Année \\
\hline & Pluie $\ldots \ldots \ldots \ldots \ldots$ & 196 & 389 & 425 & 223 & 57 & 68 & 5 & 3,7 & 55 & 3,3 & 10,1 & 4,2 & 1439 \\
\hline & Ecoulement $\ldots \ldots \ldots \ldots$ & 57 & 170 & 178 & 111 & 59,5 & 44,6 & 31,4 & 26,8 & 27,8 & 23,8 & 20,4 & 17,7 & 768 \\
\hline & 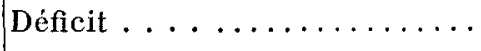 & 139 & 219 & 247 & 112 & $-2,5$ & 23,4 & $-26,4$ & $-23,1$ & 27,2 & $-20,5$ & $-10,3$ & $-13,5$ & 671 \\
\hline & $\begin{array}{l}\text { Evapotranspiration } \\
\text { effective } \ldots \ldots \ldots \ldots \ldots\end{array}$ & & & 128 & 40 & 28 & 83 & 4,5 & 5 & 49 & 4 & 10 & 5 & $\begin{array}{l}\text { env. } \\
600\end{array}$ \\
\hline & $\begin{array}{l}\text { Evapotranspiration } \\
\text { potentielle } \ldots \ldots \ldots \ldots\end{array}$ & & & 140 & 101 & 111 & 108 & 81 & 61 & 67 & 91 & 118 & 160 & \\
\hline \multirow{5}{*}{$\begin{array}{l}\stackrel{10}{\sigma} \\
\stackrel{10}{=} \\
\stackrel{20}{\infty}\end{array}$} & Pluie $\ldots \ldots \ldots \ldots \ldots \ldots$ & 110 & 262 & 128 & 360 & 549 & 63 & 10,1 & 0,2 & 4,4 & 6,1 & 6,9 & 65 & 1565 \\
\hline & Ecoulement . . . . . . . & 17,2 & 54 & 43.5 & $127, \overline{3}$ & 220 & 68,5 & 32,6 & 23,6 & 22,2 & 22,2 & 17,2 & 14,9 & 664 \\
\hline & Déficit . . . . . . . . . . . . & 92,8 & 208 & 84,5 & $232, \overline{5}$ & 329 & $-5,5$ & $-22,5$ & -23.4 & $-17,8$ & $-16,1$ & $-10,3$ & 50,1 & 901 \\
\hline & $\begin{array}{l}\text { Evapotranspiration } \\
\text { effective } \ldots \ldots\end{array}$ & 130 & 124 & 77 & 91 & 114 & 32 & 9 & 1,3 & 3,5 & 5,3 & 12 & 67 & 666 \\
\hline & $\begin{array}{l}\text { Evapotranspiration } \\
\text { potentielle ....... }\end{array}$ & 124 & 139 & 137 & 111 & 65 & 100 & & & & & & 197 & \\
\hline \multirow{4}{*}{ 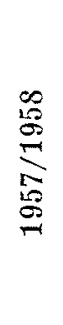 } & Pluie . . . . . . . . & 110 & 216 & 380 & 195 & 201 & & & & & & & & \\
\hline & Ecoulement . . . . . . . . & 17,4 & 52,5 & 147 & 78 & 96 & & & & & & & & \\
\hline & $\begin{array}{l}\text { Evapotranspiration } \\
\text { effective ...... }\end{array}$ & 138 & 117 & 83 & 90 & 111 & & & & & & & & \\
\hline & $\begin{array}{c}\text { Evapotranspiration } \\
\text { potentielle } \ldots\end{array}$ & & 110 & 98 & 104 & 121 & & & & & & & & \\
\hline
\end{tabular}


Le tableau ci-joint donne les résultats de la comparaison entre déficit d'écoulement mensuel sur le bassin, évapotranspiration effective mesurée sur le lysimètre et évapotranspiration potentielle mesurée sur cuve.

Trois circonstances faussent la comparaison :

- la hauteur de précipitation mensuelle n'est pas exactement la mème sur les cuves que sur le bassin, où il s'agit d'une moyenne,

- il n'y a pas de ruissellement sur ces cuves,

- le drainage est meilleur sur les cuves que sur le sol naturel.

Comme on pouvait s'y attendre, les évapotranspirations potentielles et effectives sont très voisines en saison des pluies et de lordre de 4. $\mathrm{mm} /$ jour.

I'évapotranspiration potentielle décroît à la saison sèche par suite de la baisse de la température.

Le déficit d'écoulement annuel est supérieur à l'évapotranspiration effective dans le lysimètre, probablement par suite du drainage trop fort des cuves. La rétention sur le bassin est loin d'être négligeable, la lame d'eau restituée à la saison sèche est de l'ordre de $170 \mathrm{~mm}$ en année moyenne alors que la quantité d'eau mise en réserve en saison des pluies varie peut-être de 250 à $350 \mathrm{~mm}$. Mais là, également, une, étude approfondie, jour par jour, s'impose.

Tels sont les résultats essentiels que nous avons obtenus à ce jour. Cet ensemble peut paraitre bien décousu : dans l'interprétation, nous avons couru au plus pressé. II nous faudra de nombreuses années pour exploiter, comme elle doit l'être, la masse considérable de données brutes qui ont été ainsi recueillies. Nous espérons pouvoir disposer des moyens matériels pour le faire. Mais, dès à présent, nous avons déjà acquis la conviction que cette méthode est particulièrement féconde pour l'étude approfondie des phénomènes généraux de l'écoulement.

\section{DIS CUSSI ON}

président : M. Devmé

M. le Plésident remercie M. Ronter de son exposé et le félicite d'apporter des mises au point de méthodes qui pourront etre appliquées ailleurs que dans la France d'Outre-mer.

M. Pardé souligne l'importance des résultats apportés par M. Rowrer, son sens critique qui lui permet d'apprécier les élëments d'incertitude des problèmes et les qualités de ses études qui ont grandement contribue, en une dizaine d'années, à l'édification des hydrologues sur les pays tropicaux et sur l'hydrologie en général.

Sur une remarque de $M$. le Président, $M$. Rodien précise qu'il a trouvé des évapo-transpirations potentielles de $15 \mathrm{~mm}$ en 24 heures pour les régions sud-sahariennes (latitudes supérienres à $14^{\circ}$, température de l'ordre de $45^{\circ}$ ) et de 4 à $5 \mathrm{~mm}$ en 24 heures dans les régions guinéennes à végétation équatoriale, en saison de pluies.

M. le Président pense qu'il est intéressant de rapprocher ces chiffres de ceux que M. JACQUET a trouvés pour l'évapo-transpiration potentielle en 24 heures dans le bassin du Vidourle : $4,5 \mathrm{~mm}$ en juillet et $4 \mathrm{~mm}$ en aốt.
Sur la demande de M. TMxeron', M. Ropren indique l'ordre de grandeur des intensités de précipitations décennales prises en compte dans son mémoire : Ie maximum dans les zones pour les pluies de tornade est de l'ordre de 120 a $180 \mathrm{~mm} / \mathrm{h}$ pendant des pointes de 10 minutes a un cuart d'heure; la durée totale do laverse est en génélal de 2 heures, et si hauteur totale de $100 \mathrm{~mm}$, ce qui correspond a une intensité horaire moyenue de $50 \mathrm{~mm} / \mathrm{h}$.

M. Tixenow indique que cette intensite horaire moyenne est de lordre de 20 a $30 \mathrm{~mm} / \mathrm{h}$ en lunisie.

$E_{n}$ ce qui concerne l'irrégularité des ruissellements en zone tropicale, mentionné par M. Rodier, M. 'Tixeron' dit qu'il avait remarqué cela d'après les renseignements de l'Annuaire et qu'il avait essayé d'établir une formule qui correspondait à un exposant très fort de la pluie en fonction du ruissellement : l'exposant est 3 pour la T'anisie, et 4 ou 5 pour l'A.E.F.

M. Rodrer remarque que les climats qu'il étudie sont très variés, puisque les régions intéressées s'étendent du désert à la zone équatoriale. 